Muro de la Investigación, 2020(2): julio-diciembre

ISSN: 2523-2886

Doi: https://doi.org/10.17162/rmi.v5i2.1322

\title{
EI método Singapur: sus alcances para el aprendizaje de las matemáticas
}

\author{
The Singapore Math: Its scope for learning mathematics \\ Ruth Alesshandra Tapia Reyes ${ }^{1}$, Jaimin Murillo Antón ${ }^{2}$ \\ Universidad Peruana Unión, Perú ${ }^{12}$ \\ ORCID iD: https://orcid.org/0000-0003-2281-7401 \\ ORCID iD: https://orcid.org/0000-0002-8069-0640²
}

Recibido: 08 de enero de 2020

Aceptado: 12 de junio de 2020

\begin{abstract}
Resumen
Uno de los grandes retos de las matemáticas es implementar estrategias para su aprendizaje. Esto supone un reto para los docentes hoy en día quienes, en la mayoría de los casos, no se desprenden del modelo educativo de enseñanza tradicional, cuya consecuencia es la presencia de alumnos desmotivados y con dificultades para el aprendizaje de las matemáticas. Por ello, el propósito del presente artículo es analizar los alcances que tiene el Método Singapur para la enseñanza de las matemáticas, mencionando las dimensiones, enfoques, metodología y teorías que sustentan dicho método. Este se basa en el desarrollo de pensamiento de los estudiantes para transfórmalos en ciudadanos capaces de enfrentar las exigencias de la sociedad con una actitud creativa y abierta. Asimismo, se basa sobre el enfoque Concreto, Pictórico, Abstracto (CPA). La principal conclusión obtenida es que el Método Singapur ha incrementado positivamente el aprendizaje de las matemáticas, ya que permite a los estudiantes pasar de una fase manipuladora a una fase de dibujo, y gradualmente alcanzar un nivel abstracto.
\end{abstract}

Palabras clave: Método Singapur, Enfoque CPA, matemáticas, aprendizaje

\begin{abstract}
One of the great challenges in teaching mathematics lies in to implement strategies for learning it. This is a challenge for teachers today who, in most cases, do not detach themselves from the traditional educational model of teaching - the consequence of which is the presence of unmotivated students with difficulties in learning mathematics. For this reason, the purpose of this article is to analyze the scope of the Singapore Math for teaching mathematics, mentioning the dimensions, approaches, methodology and theories that support this method. This is based on the development of students' thinking to transform them into citizens capable of facing the demands of society with a creative
\end{abstract}

${ }^{1}$ Correspondencia al autor: e-mail: ruth.tr@upeu.edu.pe; jaiminmurillo@upeu.edu.pe 
and open attitude. It is also based on the Concrete, Pictorial, Abstract (CPA) approach. The main conclusion obtained is that the Singapore Math has positively increased the learning of mathematics, since it allows students to go from a manipulative phase to a drawing phase, and gradually reach an abstract level.

Keywords: Singapore Math, CPA Approach, mathematics, learning

\section{Introducción}

Las matemáticas son consideradas fundamentales para el desarrollo intelectual de los niños; les permite ser lógicos, trabajar de manera ordenada, y preparar su mente para la crítica y la abstracción. En este caso, la dificultad de los escolares en entender las clases y, muchas veces, su bajo rendimiento académico, se deben en gran medida al método tradicional de enseñanza empleado en las escuelas.

Cabe señalar que, en el nivel inicial, es muy importante el área de matemática, porque durante esta etapa el estudiante está muy sensible para adquirir nuevos aprendizajes que serán de utilidad para su vida diaria; también aprenderá a observar, manipular, analizar, criticar y buscar soluciones a los problemas que se le presente.

Por otro lado, uno de los grandes problemas en las instituciones educativas actualmente, es que a los estudiantes se les enseña a resolver diferentes problemas de forma mecánica, sin llegar a la comprensión de lo que están resolviendo, generando en ellos aburrimiento, desmotivación $\mathrm{y}$, en el peor de los casos, rechazo hacia las matemáticas. Como resultado, se observa que la metodología de enseñanza se vuelve rutinaria, escasa de técnicas y estrategias (Quispe, 2018).

En este contexto, De La Torre (2018) afirma que gran parte de los materiales didácticos actuales están fuera del contexto y no se relacionan con el entorno o necesidad del escolar, dejando al margen su aprendizaje y sus vivencias. Aunque en los centros de educación inicial se brinda bastante contenido matemático, se descuida el orden y la secuencia, y no se presta atención a sus emociones y capacidades de aprendizaje en los más pequeños; se le da más importancia a terminar un libro; pero que no le permiten al escolar crear su propio aprendizaje, limitando sus capacidades matemáticas y sin prepararlo realmente para superar los problemas que necesita afrontar.

Hay que destacar el rol importante del maestro y que este practique un método de enseñanza que priorice la atención de las necesidades reales de los escolares, quienes se sientan parte de lo que están aprendiendo para que la enseñanza sea significativa, despertar su curiosidad, para encontrar nuevos caminos de soluciones durante las 
experiencias de su diario vivir. Por ello, este Método Singapur persigue cambiar la perspectiva en la formación y la enseñanza de las matemáticas; ha tenido gran aceptación a nivel mundial. A continuación, se describirá este método y sus alcances para la educación inicial.

\section{Método Singapur}

\section{Conceptos}

El Método Singapur es una aplicación de pedagogía de matemática que se construye sobre la base de la investigación. Es el resultado de un estudio internacional de los mejores métodos de enseñanza. Jerome Bruner, Zoltan Dienes y Richard Skemp son sus principales representantes. Este método no se orienta en la memorización, la enseñanza de procedimientos o la aplicación de fórmulas (Delgado, Mayta, \& Alfaro, 2018); antes bien, se basa sobre estrategias, las cuales ayudan a enseñar de una manera diferente. Dicho método busca docentes que trabajen en conjunto con sus estudiantes. Lo principal es que se escuchen las ideas de los estudiantes, a fin de que, en vez de llegar a la memorización, se logre la resolución de cada problema (Hilaquita, 2018).

Como una estrategia concreta, el Método Singapur favorece el desarrollo de procesos, actitudes y habilidades que fomentan el pensamiento matemático; se representa por hacer de la resolución de problemas un foco del proceso (Juarez \& Aguilar, 2018). Esta metodología difiere con la demasiada memorización y cálculo de la enseñanza tradicional; sugiere enseñar a los estudiantes para solucionar problemas por sí mismos; también para aprender a pensar. En totalidad, las clases de matemática del Método Singapur se dan inicio de la misma forma: el docente propone un problema y los estudiantes deliberan sobre cómo llegar a una solución. Este método implica llegar a una misma solución por diferentes caminos, teniendo un aprendizaje basado en problemas.

Según el Ministerio de Singapur (2012), el objetivo general del currículo del área de matemática es garantizar que todos los estudiantes puedan alcanzar un nivel de dominio que les ayudará para beneficio de la vida, por lo que los objetivos generales de la educación matemática en Singapur están enfocados en lograr que los estudiantes apliquen y obtengan destrezas y conceptos matemáticos, desarrollen habilidades cognitivas y meta cognitivas, a través del enfoque de resolución de problemas y desarrollen actitudes positivas hacia las matemáticas (García Morales, De la Carrera Fol, y Muela Fernández, 2013). Según Debrán (2015), esto será posible si se usa la estructura pentagonal que enlaza el desarrollo de conceptos, habilidades, procesos matemáticos, 
meta cognición y actitudes esenciales para el aprendizaje, cuyo eje central es la resolución de problemas en contextos significativos.

\section{Teorías que sustentan al Método Singapur}

\section{Jerome Bruner-enfoque CPA}

Singapur apoya su metodología sobre la teoría de Jerome Bruner: para alcanzar una buena enseñanza se debe adquirir un completo conocimiento conceptual; los estudiantes deben vivir tres procesos: concreto, pictórico, abstracto (Hilaquita, 2018).

Concreto. Los estudiantes utilizan material concreto, real, palpable y cercano; objetos de uso durante la vida cotidiana: bloques, fichas, piezas, cubos, pelotas, o cualquier otro objeto que a los estudiantes los motive.

Pictórico. Se debe inducir al estudiante para construir una representación gráfica de las relaciones entre cantidades o los procesos matemáticos subyacentes: imágenes y dibujos que le ayuden a resolver un problema.

Abstracto. Conecta esos procesos con algoritmos y formulaciones de la matemática más abstracta, alcanzando la comprensión del concepto trabajado, utilizando símbolos y signos.

Partiendo de esta premisa, se espera que los estudiantes identifiquen la relación entre los datos y la incógnita del problema, comprenderla mejor y resolverla.

\section{Currículo en espiral}

El currículo debe organizarse de forma espiral, se debe trabajar periódicamente los mismos contenidos; los estudiantes vuelven a desarrollar las ideas principales y van profundizando la comprensión; por eso el proceso de aprendizaje logra gran significación, pues no se basa en saturar al niño con conceptos, sino busca su real comprensión y entendimiento de forma progresiva (Gonzáles, 2015).

\section{Zoltan Dienes - Variación Sistemática}

Por su lado, Calderon (2014) dice que otro fundamento teórico que sustentan el Método Singapur es la Variación Sistémica de Zoltan Dienes, la cual, apoyándose sobre los planteamientos teóricos de Piaget y Brunner, ha difundido una mirada de lo que es y 
lo que debe ser la educación matemática durante el tiempo y ha incidido en este quehacer en diversos países del mundo. Dicha teoría sostiene que las estructuras matemáticas sean enseñadas desde los primeros grados empleando múltiples ejecuciones: juegos, materiales manipulativos, cantos, bailes, etc. Zoltan ha sido el inventor de materiales: los bloques multibase, los bloques lógicos y otros materiales para el área de algebra, por ejemplo. Por su parte, las investigaciones de Brunner sustentan que personas distintas abordan un mismo problema de modo diferente, lo que significa que para el aprendizaje hay que tener en cuenta: la estructura lógica del contenido y la estrategia mental que cada persona usa. De esta forma, Dienes estudió algunos principios que, respecto a la enseñanza de la matemática en los primeros grados, se debe tener en cuenta:

a) Principio de la constructividad. El aprendizaje de la matemática es una actividad constructiva perseverante de los conceptos, está antes que el análisis de la formación de conceptos matemáticos; el estudiante debe construir y elaborar dichos conceptos.

b) Principio dinámico. Según este principio, los estudiantes revelan experiencias, las cuales son concretas, mediante el uso del material adecuado y los juegos.

c) Principio de la variabilidad de perspectiva. El estudiante tiene varias y diversas formas de percibir; las diferencias son personales, individuales, por la diversidad de materiales, formas perceptivas, características relevantes y diferentes.

\section{Richard Skemp}

Para Arias-Huerta et al. (2017), otro de los teóricos que aportó para esta metodología fue Richard Skemp, quien analizó la diferencia entre comprensión relacional (saber qué) y comprensión instrumental (saber hacer). Estos dos tipos no siempre van unidos. Los estudiantes deben aprender las matemáticas con comprensión, construyendo activamente los nuevos conocimientos a partir de la experiencia y los conocimientos previos. Los indicadores de idoneidad didáctica de procesos de enseñanza y el aprendizaje de las matemáticas, también las orientaciones curriculares consideran que el aprendizaje significativo supone comprender y ser capaz de aplicar los procedimientos, conceptos y procesos matemáticos.

a) Comprensión instrumental. Dentro de este criterio, el autor deja en la capacidad de resolver una operación matemática utilizando las reglas generales, lápiz y papel. Para llegar a la resolución de problemas, los estudiantes necesitan el 
docente para aprender los pasos. La comprensión instrumental apresura el proceso de enseñanza-aprendizaje, este logro se basa sobre la memorización de fórmulas que el estudiante debe realizar y teniendo la respuesta más confiable y rápida.

b) Comprensión relacional. Se basa en tener la capacidad de explicar el conocimiento seguido para resolver el problema. Los mismos estudiantes crean una estructura conceptual, para que puedan crear sus propias estrategias al resolver problemas, aunque no se encierran en una sola manera, ya que los estudiantes, la mayoría de veces, toman conciencia de que hay diferentes posibilidades. Dichos aprendizajes son más fáciles para recordar, ellos mismos aportaron para la construcción de la interacción y la colaboración, haciendo que estos conocimientos sean parte de un todo conectado. Por último, refuerza el rol de protagónico que tiene el estudiante durante el proceso de enseñanza-aprendizaje; aunque le tome un poco más de tiempo en llegar a una respuesta; el objetivo no es obtener la recompensa: el resultado del problema, sino desarrollar el pensamiento lógico matemático, apoyado sobre la realidad y sobre conceptos cercanos al estudiante.

\section{Modelo de enseñanza del Método Singapur}

Singapur apunta a los estudios de Oviedo \& Panca (2017), quienes enfatizan que un buen plan de enseñanza debe conectar los objetivos del aprendizaje con los tipos de actividades a desarrollar con los alumnos. Por ello, este modelo de enseñanza de matemáticas estructura sus actividades según el esquema: asentamiento, trasferencia, consolidación y comprensión.

Para lograr un aprendizaje exitoso de las matemáticas se debe centrar en el aprendizaje con comprensión; así los estudiantes recordarán los procedimientos y podrán razonar solo si aprenden comprendiendo lo que hacen; empleando las matemáticas para la resolución de problemas; es el objetivo principal del currículo de matemática en Singapur. Fundamentar la enseñanza a los estudiantes, en conocer sus intereses y su experiencia e involucrarlos en el aprendizaje reflexivo y activo.

En este sentido, Edge (2014, en Bastas, Olea, \& Trincado, 2015) encontró un esquema, el cual lo actualizó y hoy en día ayuda a acomodar con mayor facilidad los procesos necesarios, para dar una buena clase utilizando el Método Singapur a través de tres actividades: comprensión, consolidación y transferencia, las cuales son las etapas de inicio, desarrollo y cierre de las clases ejecutadas en nuestro país.

\section{Comprensión}


La primera etapa del modelo de enseñanza del Método Singapur implica la aproximación inicial que tiene el estudiante hacia un concepto determinado, utilizando material concreto o pictórico (iniciación), que es el paso al mundo abstracto a través de la utilización de otros modelos concretos/pictóricos o enunciados verbales (abstracción), a fin de ayudar al estudiante a promover e identificar un patrón determinado (esquematización). Esto incluye el principio de la clase y se subdivide en tres secciones:

a) Iniciación. Se da a conocer a los estudiantes un concepto determinado: base para un nuevo conocimiento sobre estructuras ya existentes, usando el bagaje cultural propio del alumno(a) y sus previos conocimientos sobre el concepto para "atraerlo" al tema, siendo importante darle el estímulo adecuado, para que se realice mediante el uso de materiales concretos o pictóricos.

b) Abstracción. Los estudiantes deben ser capaces de integrar el concepto aprendido, mediante la ejecución general de los ejemplos proporcionados por el docente.

c) Esquematización. La etapa final de comprensión incluye adquirir el concepto del estudiante, mediante la identificación de patrones, relaciones o práctica en su vida diaria.

\section{Consolidación}

La segunda etapa del modelo de enseñanza, según las directrices del Método Singapur, ocurre desde el instante en que el docente tiene la certeza de que los estudiantes han podido entender los conceptos dados en la etapa anterior. El propósito principal de consolidación es ayudar a los estudiantes a recordar los hechos y las destrezas afiliadas al concepto, practicando su dominio mediante actividades lúdicas.

\section{Transferencia}

Posteriormente a la adquisición del contenido por el estudiante, expresada en la denominación y en aplicación del concepto, se llega a la etapa de transferencia. Se proponen diferentes tareas o situaciones, los estudiantes tienen la oportunidad de aplicar sus conocimientos mediante el uso heurístico de sus habilidades y capacidades, en circunstancias de usos diarios de la matemática: el fin último de toda clase con el Método Singapur. En esta última etapa, los conocimientos se transfieren, se aplican en la solución de problemas de rutina o no, en proyectos de investigación o simplemente en preguntas abiertas, las cuales despierten, en algún grado, el interés en la asignatura.

\section{Evaluación}


No es el fin último del modelo de enseñanza con el Método Singapur, no obstante, es central e integral en el proceso de enseñanza-aprendizaje. Esta etapa se presenta durante el desarrollo del modelo mediante evaluaciones de diagnóstico (al comienzo de la etapa de comprensión, con el objetivo de identificar conocimientos previos y nivel inicial de los estudiantes con relación a un tema determinado), formativa (mediante el desarrollo de las diferentes actividades durante la enseñanza, con las participaciones escritas y orales de los estudiantes, siendo ayudada por rúbricas, listas de cotejo, instrumentos similares de evaluación), y sumativa (pruebas globales, cuyo objetivo es reconocer la consolidación de los aprendizajes esperados, normalmente realizados a través de una prueba escrita).

\section{Componentes del método Singapur}

Según Fernandez (2015), Singapur desarrolló un currículo de matemática sobre la base de cinco componentes, que enfatizan la comprensión de conceptos, habilidades y procesos matemáticos; además, otorga especial importancia a las actitudes y la metacognición. Estos cinco componentes que están interrelacionados son:

Conceptos. Para la comprensión profunda y generar sentido a las ideas matemáticas, sus conexiones y aplicaciones, los estudiantes viven variedad de experiencias, actividades prácticas, además usan herramientas tecnológicas.

Habilidades. Sirven para el aprendizaje y la aplicación de las matemáticas, cuyas habilidades matemáticas son usadas y practicadas, comprendiendo los principios subyacentes y los procedimientos.

Procesos. Refieren las habilidades de proceso: adquisición y aplicación, razonamiento, comunicación y conexiones, habilidades de pensamiento, métodos de investigación, aplicación y modelamiento.

Metacognición. Refiere la toma de conciencia y la capacidad de controlar los procesos: selección y uso de estrategias (monitoreo del pensamiento y la autorregulación del aprendizaje). Para desarrollar estrategias meta cognitivas y aprender cómo y cuándo utilizarlas, los estudiantes resuelven problemas no rutinarios, debaten sobre las posibles soluciones, pensar en voz alta y reflexionar sobre lo que están haciendo, analizar los procedimientos y realizar cambios cuando sea necesario.

Actitudes. Refieren los aspectos afectivos del aprendizaje de matemáticas.

Los cinco componentes integran el aprendizaje de las matemáticas y la resolución de problemas, mediante el método Singapur, con la finalidad de ayudar a los docentes a 
focalizar estos componentes en sus alumnos, promoviendo la creatividad, el desarrollo de las habilidades, el pensamiento creativo, las capacidades, el razonamiento, la comprensión conceptual y la conciencia.

\section{Alcances del método Singapur}

\section{Implementación del método Singapur en Chile}

Angulo, Castillo y Niño (2016) refieren que luego de la firma de convenio entre Singapur y Chile, el Centro Felix Klein trabajó el Método Singapur. Dinko Mitrovich, subdirector de dicho centro, refirió los desafíos y el impacto del método, cuyo trabajo de adaptación es coherente con los planes y programas del Ministerio de Educación. El objetivo fue replicar óptimamente los principios del método Singapur y quedaron a la vista las diferencias significativas entre estos dos países: en Singapur, los niños dan los primeros pasos de la multiplicación en primer año básico; en Chile, comienza en tercer año; en Singapur trabajan en el segundo año; en Chile, uno o dos años después. En el plan curricular chileno, se enseñan en general más con ilustraciones; los niños de Singapur, en cambio, desde pequeños se acostumbran a trabajar con estos objetos de estudio, y aprenden a construirlos y usarlos, impresionando la cantidad y complejidad de los ejercicios que los niños realizan desde muy pequeños (Morales Espinoza, 2012).

Se realizó una prueba y aplicaron dicho proyecto en más de 100 escuelas de distintas regiones del país. A pesar de las condiciones, solo contaron con material y con profesores, quienes no habían sido capacitados; el Método Singapur ya había tenido leves avances respecto de la "escuelas de control". Este es un indicador importante de la eficacia del método, porque cuando se introduce una metodología, y según se ha visto a nivel internacional, lo normal es que al principio se produzca un leve retroceso; se explica principalmente debido a que cuando el profesor ya está acostumbrado a una metodología y se introduce en una nueva, se desestabiliza un poco. En un sistema educacional bastante segregado, que los colegios particulares chilenos de excelencia lo estén utilizando, es una señal bastante importante frente a la necesidad de muchos establecimientos de tener un referente de enseñanza de la matemática y en esto el Método Singapur lo está resolviendo. "Nosotros recibimos muchos llamados solicitando capacitaciones y asesorías, tantos que no damos abasto por ser un centro pequeño y vemos que se está generando bastante entusiasmo así mismo hoy muchos colegios están utilizando el Método Singapur o quieren comenzar a utilizarlo”, concluyó Mitrovich subdirector del centro Felix Klein.

\section{Experiencia del Método Singapur en Colombia}


La Secretaria de Educación de Barranquilla, Karen Abudinen, anunció que 50 colegios de Barranquilla implementarán el método Singapur, cuya incorporación se realizó el 2012, generando un beneficio para 2.000 estudiantes; actualmente son beneficiados más de 30.000, también se han beneficiado más de 15.000 docentes, logrando un ascenso de 7 puntos, quedando Barranquilla en una posición destacada. El año referido, se trabajó con los grados de $1^{\circ}$ al $5^{\circ}$, en 18 colegios que funcionaron en la condición de pilotos, luego se incrementó el número a 32, trabajando con los grados $1^{\circ} \mathrm{y}$ $2^{\circ}$. El año 2014, se obtuvieron resultados muy destacados sobre sus competencias evaluadas, el $80 \%$, el $77 \%$ y el $62 \%$ de los estudiantes de $1^{\circ}$, el $2^{\circ}$ y el $3^{\circ}$ grado, respectivamente (Mamani, 2018).

\section{Experiencia del Método Singapur en España}

En la investigación de Fernandez (2015) se aplicó el método Singapur en tres grados diferentes, en la cual concluyó que utilizó en su aula dicha metodología de manera grata. Tras la aplicación de este método de trabajo, observó un mayor interés de los estudiantes en las matemáticas, que ya tenían interiorizado. El trabajo en grupo resulta extraño para el alumnado, debido a que en el área matemática se suele trabajar de forma individual. Al utilizar este método, se comprueba que sí se pueden trabajar las matemáticas de forma grupal.

\section{Conclusiones}

Este estudio confirma que el Método Singapur es eficiente para la enseñanza de las matemáticas, cuyo beneficiario es el niño, quien inicia el proceso exponiendo sus conocimientos previos, sus experiencias, sus fortalezas y limitaciones. También constituye un modelo innovador de enseñanza, tanto en el nivel primario como secundario. Permite la adaptación de los niños, cuyos escenarios son nuevos y diferentes.

Además, se ratifica que este método ha superado el método tradicional, que se ha quedado solamente en la memoria de reglas, ecuaciones, pasos y procedimientos. Enfoca procesos, actitudes, habilidades cognitivas y metacognitivas, durante la resolución de los problemas. Asimismo, fomenta la autoconfianza, la comunicación, la colaboración, el trabajo colectivo y colaborativo, el desarrollo de hábitos de aprendizaje, el sentido de auto dirigido, establecer sus propios objetivos y competencias, mirando el futuro de ciudadanos para la sociedad. 


\section{Referencias}

Arias Huerta, T., Arrunátegui Valencia, C., Julca Salazar, L., \& Zúñiga Torres, K. (2017). Mejora del proceso de enseñanza - aprendizaje de las competencias matemáticas tempranas mediante la aplicación del método de Singapur, las clases eurítmicas y los grupos interactivos en los niños y niñas de 4 años del aula "tulipanes" de la institución educativa (Tesis de licenciatura). Universidad Pedagógico Nacional Monterrico. Lima, Perú.

Bastas, A., Olea, D., \& Trincado, N. (2015). Efectividad del método Singapur en el desempeño académico de los estudiantes de cuarto año básico en la asignatura de educación matemática. (Tesis de Licenciatura). Universidad Andres Bello, Santiago, Chile. Recuperado de: https://doi.org/10.1145/3132847.3132886

Calderon, P. (2014). Percepciones de los y las docentes del primer ciclo básico, sobre la implementación del método Singapur en el colegio Mario Bertero Cevasco de la comuna de isla de Maipo. (Tesis de maestria). Universidad de Chile, Santiago, Chile.

De La Torre, V. (2018). Eficacia del programa "Jugando y pensando voy avanzando" en el desarrollo de la noción del número en niños de 5 años de la I.E.P "Estrellitas" Ugel $N^{\circ} 06$ Ate,2018. (Tesis de licenciatura). Universidad Peruana Unión, Lima, Perú.

Delgado, M., Mayta, E., \& Alfaro, M. (2018). Efectividad del "Método Singapur" en la Resolución de problemas matemáticos en estudiantes del tercer grado de primaria de una institución educativa privada del distrito de Villa El Salvador. (Tesis de maestria). Pontificia Universidad Católica del Perú, Lima, Perú.

Fernandez, D. (2015). El método Singapur aplicado a la enseñanza de fracciones. (Tesis de licenciatura) Universidad de Valladolid, Valladolid, España. Recuperado de: https://doi.org/10.1103/PhysRevB.79.193407

García Morales, P., De la Carrera Fol, R., y Muela Fernández, Á. (2013). Método Singapur. Sites.cardenalcisneros.es.

Gonzáles, L. (2015). Efecto del método Singapur en el desarrollo de competencias matemáticas para niños de $3^{\circ}$ de básica primaria. (tesis de maestría) Universidad de la Costa CUC. Barranquilla, Colombia. Recuperado de: https://doi.org/10.1145/3132847.3132886

Hilaquita, V. (2018). Método Singapur en la resolución de problemas matemáticos en los estudiantes del quinto grado de educación primaria de la institución educativa 
mercedario san pedro pascual de la ciudad de Arequipa 2018. (Tesis de maestria). Universidad Nacional de San Agustin, Arequipa, Perú.

Juarez, M. del R., y Aguilar, M. (2018). El método Singapur, propuesta para mejorar el aprendizaje de las Matemáticas en Primaria. Números, 98(12), 75-86.

Lucila-Angulo, G., Castillo-Echeverry, J., y Niño-Pérez, S. (2016). Propuesta de implementación del método Singapur para enseñar las matemáticas en niños de segundo de primaria en el gimnasio los Arrayanes. Universidad de La Sabana. (tesis para optar la especializacion en gerencia educativa) Universidad de la Sabana, Cundinamarca, Colombia.

Mamani, E. (2018). Eficacia del método Singapur para mejorar las competencias matemáticas de los estudiantes del primer grado de educación primaria de la institución educativa bellavista del distrito de Juliaca. (tesis de doctorado). Universidad Nacional de San Agustin, Arequipa, Perú.

Morales Espinoza, N. (2012). Método Singapur: Descripción de su Implantación. Factores facilitadores y/o obstaculizadores. Una experiencia del profesorado de primer ciclo básico en una escuela municipal en la ciudad de Valdivia. (tesis de maestría). Universidad de la Frontera, Temuco, Chile.

Oviedo, M. A., \& Panca, G. C. (2017). Influencia del Método Singapur en la resolución de problemas aditivos en los estudiantes de segundo grado del nivel primaria de la Institución Educativa 40199 de Ciudad Mi Trabajo del Distrito de Socabaya Arequipa, 2017. (Tesis de licenciatura). Universidad Nacional de San Agustín de Arequipa, Arequipa, Perú.

Quispe, J. (2018). Programa "Matemática con la naturaleza" para desarrollar las nociones matemáticas en estudiantes de 5 años del nivel inicial de la I.E $N^{\circ} 659$ “María Montessori” Pisquicocha, Cotaruse, Aymaraes, Apurímac, 2018. (tesis de licenciatura). Universidad Peruana Union, Lima, Perú.

Rivas, C. Y. (2018). Nivel de desarrollo lógico matemático en los niños de 5 años de la I.E. Innova Schools, Canta Callao, 2018. Universidad Cesar Vallejo. 